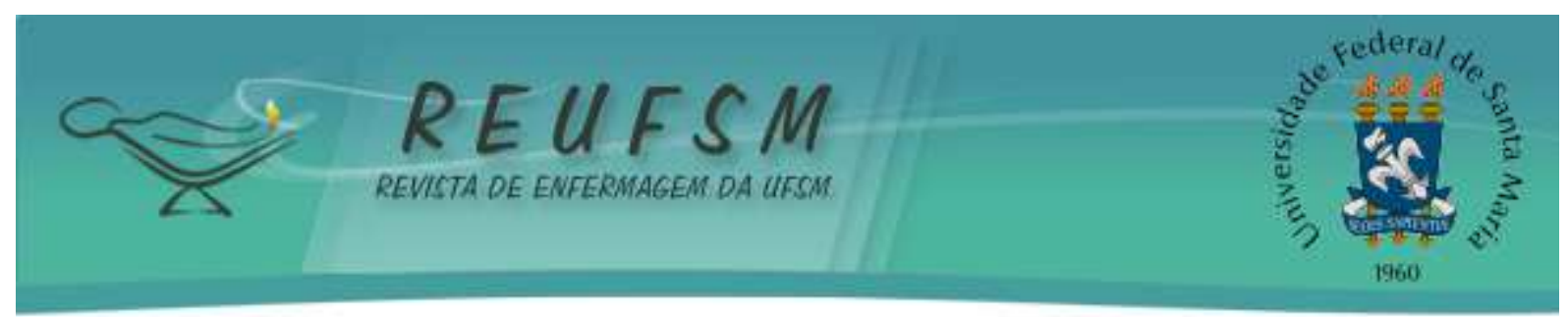

\title{
RESULTADOS DE PARTOS DOMICILIARES PLANEJADOS ASSISTIDOS POR ENFERMEIRAS OBSTÉTRICAS
}

\section{RESULTADOS DE LOS PARTOS DOMICILIARES PLANEADOS Y ASISTIDOS POR ENFERMERAS OBSTÉTRICAS}

\section{OUTCOMES OF PLANNED HOME BIRTHS ASSISTED BY NURSE MIDWIVES}

\author{
Simone Silva dos Santos ${ }^{1}$ \\ Lara Mabelle Milfont Boeckmann ${ }^{2}$ \\ Ana Cyntia Paulin Baraldi ${ }^{3}$ \\ Manuela Costa Melo ${ }^{4}$
}

Doi: $10.5902 / 2179769228345$

RESUMO: Objetivo: descrever os resultados maternos e neonatais de partos domiciliares planejados assistidos por enfermeiras obstétricas. Método: estudo descritivo, exploratório, retrospectivo, documental e de caráter quantitativo. A amostra envolveu 99 prontuários de mulheres, em 2015, oriundos de três equipes de parto domiciliar do Distrito Federal, Brasil. Utilizou-se o Statistical Package for Social Science versão 20.0, obtendo-se uma análise estatística descritiva. Resultados: verificou-se média de idade de 31,6 anos; ensino superior completo $(87,9 \%)$; união estável $(90,9 \%)$; exerciam atividade remunerada $(78,8 \%)$; eram primíparas ou não tinham parto normal anterior $(63,6 \%)$; todas adotaram posições verticalizadas; $87,5 \%$ dos recém-nascidos tiveram contato pele a pele e amamentação precoce; todos tiveram clampeamento tardio do cordão umbilical; e $98,7 \%$ apgar $\geq 7$ no $5^{\circ}$ minuto. A minoria das mulheres $(21,1 \%)$ e dos recém-nascidos $(1,2 \%)$ foram transferidos para o hospital, porém sem complicações. Conclusão: os resultados evidenciam uma assistência de qualidade e segura realizada por enfermeiras obstétricas, corroborando outros achados científicos.

Descritores: Parto domiciliar; Enfermagem obstétrica; Parto normal; Parto humanizado; Assistência perinatal.

ABSTRACT: Aim: to describe maternal and neonatal outcomes of planned home births assisted by nurse-midwives. Methods: descriptive, exploratory, retrospective, documental and quantitative study. The sample included 99 records from three home birth teams from Brasília, Brazil, in 2015. The Statistical Package for Social Science version 20.0 was applied to obtain a descriptive statistical analysis. Results: women's mean age was 31.6 years, $87.9 \%$ had higher education, $90.9 \%$ were married, $78.8 \%$ were engaged in remunerated activities, $63.6 \%$ were primiparous or had no previous delivery, all of them adopted vertical position, $87.5 \%$ of newborns were placed skin-to-skin with their mothers and initiated breastfeeding, all the newborns had delayed umbilical cord clamping and 98.7\% received 5th minute APGAR $\geq 7$.

\footnotetext{
${ }^{1}$ Enfermeira Obstétrica da Equipe Olhar Sereno - Parto Domiciliar Planejado. Especialista em Enfermagem Obstétrica pelo Programa de Residência Multiprofissional em Rede do Distrito Federal. Brasília, DF, Brasil. Email: eo.simonesilva@gmail.com.

2 Docente da Escola Superior de Ciências da Saúde. Doutora em Enfermagem pelo PPGEnf/UnB. Secretaria de Estado de Saúde do Distrito Federal (SES-DF). Brasília, DF, Brasil. E-mail: laramilfont@gmail.com.

${ }^{3}$ Enfermeira Obstétrica e Neonatologista da Luz de Candeeiro - Parto Domiciliar Planejado. Mestre em Ciências da Saúde pela Enfermagem de Ribeirão Preto. Brasília, DF, Brasil. E-mail: anacyntiapb@gmail.com.

${ }^{4}$ Docente da Escola Superior de Ciências da Saúde. Doutora em Enfermagem pelo PPGEnf/UnB. Secretaria de Estado de Saúde do Distrito Federal (SES-DF). Brasília, DF, Brasil. E-mail: melomanuela91@ gmail.com
} 


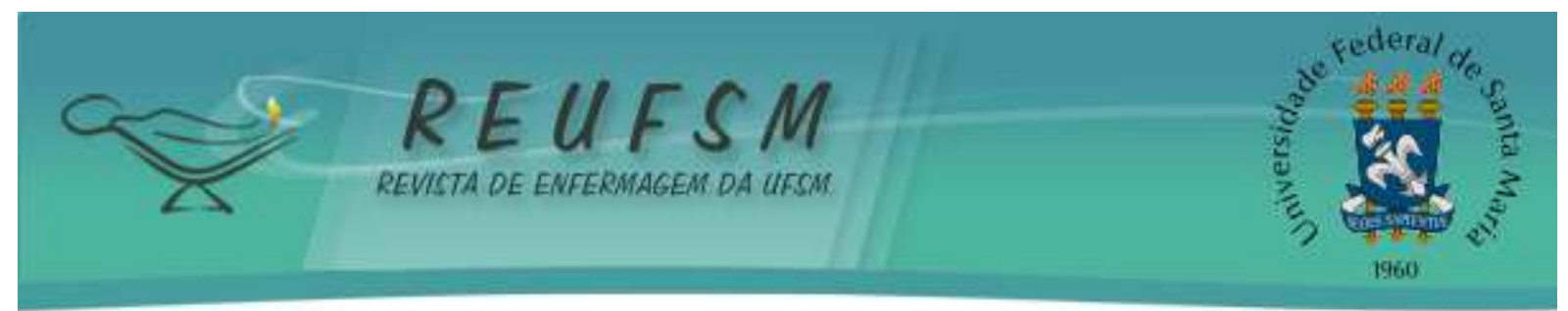

$21 \%$ of women and $1.2 \%$ of newborns were transferred to the hospital, without any unfavorable condition. Conclusion: outcomes indicated that planned home births attended by nurse midwives provide a qualified and safe assistance, confirming other scientific findings.

Descriptors: Home childbirth; Obstetric nursing; Natural childbirth; Humanizing delivery; Perinatal care.

RESUMEN: Objetivo: describir los resultados maternos y neonatales de partos domiciliarios planificados y asistidos por enfermeras obstétricas. Método: estudio descriptivo, exploratorio, retrospectivo, documental y de carácter cuantitativo. La muestra analisada se basó en 99 histórias clínicas de mujeres, de 2015, procedentes de tres equipos de parto domiciliar del Districto Federal, Brasil. Se utilizó el Statistical Package for Social Science versión 20.0, obteniéndose un análisis estadístico descriptivo. Resultados: se verificó una media de edad de 31,6 años; educación superior completa (87,9\%); unión estable (90,9\%); que ejercían actividad remunerada (78,8\%); eran primíparas o no tenían parto normal anterior (63,6\%); todas adoptaron posiciones verticalizadas; $87,5 \%$ de los recién nacidos tuvieron contacto piel a piel y lactancia precoz; todos tuvieron clampeo tardio del cordón umbilical; y el 98,7\% recoger $\geq 7$ en el quinto minuto. La minoría de las mujeres $(21,1 \%)$ y de los recién nacidos (1,2\%) fueron trasladados al hospital, sin complicaciones. Conclusión: los resultados evidencian una asistencia de calidad y segura realizada por enfermeras obstétricas, corroborando con otros descubrimientos científicos.

Descriptores: Parto domiciliário; Enfermería obstétrica; Parto normal; Parto humanizado; Atención perinatal.

\section{INTRODUÇÃO}

A assistência ao parto e nascimento vem sendo tema de discussões em diversos países e têm repercutido em muitas mudanças. No passado, essa assistência era prestada por parteiras, predominantemente no domicílio, com acesso limitado aos recursos e representava, em sua essência, um evento familiar. Ao passar dos anos e com o rápido avanço tecnológico e industrial, o parto se institucionalizou, centrando na figura do médico, e sendo visto como um processo patológico, medicalizado e intervencionista. ${ }^{1} \mathrm{O}$ movimento atual busca o resgate do parto como um evento familiar, o protagonismo e a autonomia da mulher; sendo o domicílio novamente um local para o parto, porém como uma opção dotada de informações, planejamento e recursos. ${ }^{2}$

O parto domiciliar planejado (PDP) é habitual em países desenvolvidos como, por exemplo, no Reino Unido, Canadá, Austrália e Holanda, onde há o incentivo dessa modalidade de assistência, não só por apresentar menor custo ao sistema de saúde, mas pela existência de evidências científicas constatando sua segurança. ${ }^{3}$ Apresenta desfechos materno-neonatais favoráveis, com menor índice de cesariana, de intervenções desnecessárias, neonatos com menor necessidade de reanimação, entre outros. ${ }^{4-5}$ 


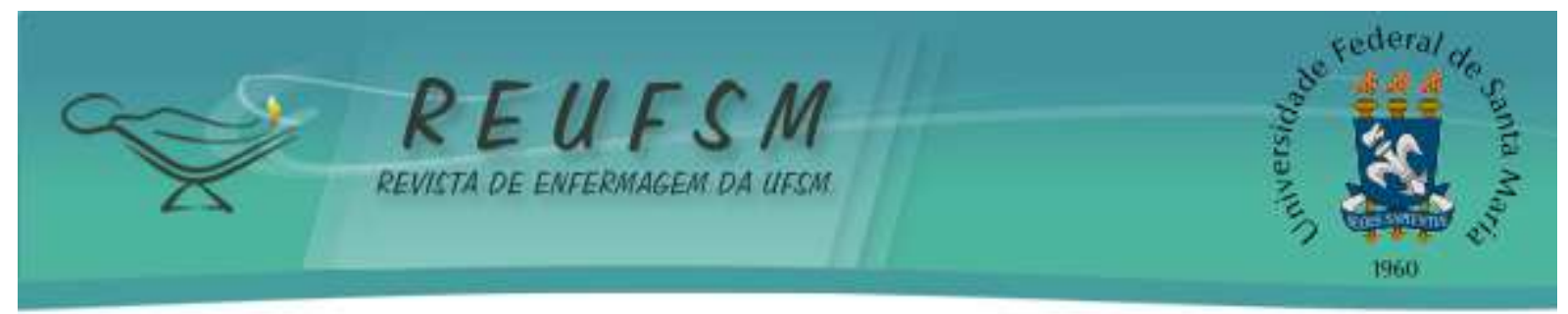

No Brasil, os partos têm ocorrido, predominantemente, nas instituições hospitalares e ainda que este local seja considerado um ambiente seguro, é evidente que a atual assistência obstétrica vivencia uma dicotomia com relação às recomendações da Organização Mundial da Saúde (OMS) e as práticas realizadas. Além das altas taxas de cesarianas (em torno de 57\%), tem-se uma assistência em grande parte inadequada, medicalizada e intervencionista. Embora haja políticas e programas que tangem as boas práticas de atenção ao parto e nascimento, como a Política de Humanização do Parto e Nascimento, a Rede Cegonha, dentre várias diretrizes do Ministério da Saúde e ações da Agência Nacional de Saúde, pouco se tem avançado. ${ }^{6-8}$

A mais recente publicação do Ministério da Saúde sobre as diretrizes para o parto normal não recomenda o PDP em nulíparas e afirma que as evidências oriundas de outros países não necessariamente são aplicáveis ao Brasil. No entanto, recomenda o PDP em multíparas de risco habitual, desde que seja assegurado o acesso em tempo hábil e oportuno a uma maternidade, se houver necessidade de transferência. Embora as evidências internacionais apontem o PDP como seguro, ele é contestado por vários profissionais, os quais alegam ser perigoso mesmo em situações de risco habitual, e acreditam que o hospital é o único lugar seguro. ${ }^{2-6}$

No Distrito Federal, dados obtidos no Sistema de Informações sobre Nascidos Vivos (SINASC), indicam um aumento na ocorrência de partos em domicílio, passando de 47 em 2011, 151 em 2013, para 213 em 2016. Apesar desses dados não diferenciarem o PDP dos partos desassistidos ou acidentais, eles requerem atenção, pois tem se evidenciado um constante aumento. Outro fator relevante é que grande parte dessas ocorrências refere-se às mulheres consideradas instruídas, com escolaridade $\geq 12$ anos, correspondendo a $6,4 \%$ dos partos domiciliares de 2011, 50,3\% em 2013, e 65,7\% em 2016, o que pode indicar o aumento dessa opção pelas famílias na capital do país. ${ }^{9}$

Em relação aos profissionais habilitados no atendimento ao PDP, destaca-se a enfermeira, a qual possui respaldo legal para atuar na assistência ao pré-natal e parto normal de risco habitual. A enfermeira obstétrica tem sido reconhecida por favorecer o uso criterioso de intervenções e a redução de procedimentos desnecessários, aumentando a satisfação da mulher e a qualidade na assistência. ${ }^{6,10-11}$ Estudo aponta maior autonomia de enfermeiras obstétricas em PDP, e respaldo científico pelo emprego das Práticas Baseadas em Evidências de forma integral. ${ }^{2} \mathrm{Na}$ assistência domiciliar, essas especialistas devem ter habilidades específicas para realizar uma avaliação contínua, identificando precocemente os sinais de 


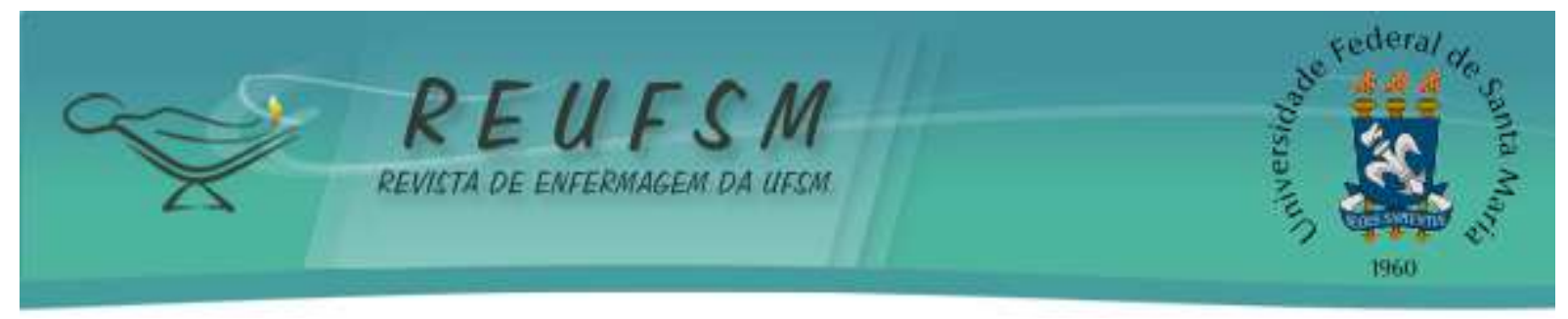

riscos, oferecendo suporte básico de vida e estabilizando as mulheres e os neonatos, até a transferência hospitalar, nos casos de intercorrências. ${ }^{6}$

Apesar do reconhecimento e incentivo da atuação dessa profissional, da notável expansão do PDP em todo território nacional e da crescente busca dessa modalidade de assistência por mulheres que desejam resgatar e protagonizar o processo de gestar, parir e cuidar de seus filhos, existem desafios relacionados a essa escolha. ${ }^{1,10}$ Estudos em âmbito nacional favorecem o enfrentamento desses desafios, como a desconstrução de crenças préconcebidas e sem sustentação científica que consideram o PDP como um retrocesso e com índices de morbimortalidade ditos maiores que nas instituições hospitalares, fatores que estigmatizam as famílias que optam por essa forma de assistência. ${ }^{2,12}$

Neste sentido, propôs-se a seguinte questão de pesquisa: quais os resultados maternos e neonatais de partos domiciliares planejados assistidos por enfermeiras obstétricas no Distrito Federal, Brasil? O conhecimento desses resultados possibilita inferir sobre a assistência prestada por essas profissionais, a segurança e as complicações envolvidas nessa modalidade o que, associado ao aumento do público que busca esse tipo de atendimento e da escassez de produção científica que aborde essa temática, justifica a realização da presente pesquisa.

Diante do exposto, o objetivo do estudo foi descrever os resultados maternos e neonatais de partos domiciliares planejados assistidos por enfermeiras obstétricas.

\section{MÉTODO}

Trata-se de um estudo descritivo, exploratório, retrospectivo, documental e de caráter quantitativo. A amostra totalizou 99 prontuários, correspondente a 100\% das mulheres e seus recém-nascidos (RNs) atendidos no período de janeiro a dezembro de 2015, por três equipes de PDP do Distrito Federal, denominadas como equipe A, B e C.

Ainda não se tem dados documentados sobre todas as equipes que atendem PDP na capital do Brasil. As três equipes foram selecionadas por concordarem participar da pesquisa e estarem atuando nessa modalidade no período estudado. Cada equipe é formada por, pelo menos, duas enfermeiras obstétricas. São serviços privados e autônomos, que atendem à demanda espontânea proveniente do Distrito Federal e possuem ainda uma rede de médicos obstetras que podem ficar de sobreaviso para necessidade de transferências, a depender do planejamento e opção feita pela gestante. Dentre os diversos serviços oferecidos, destaca-se o pré-natal sensível com foco no PDP, assistência ao trabalho de parto e parto no domicílio e consultas ao binômio durante o pós-parto. 


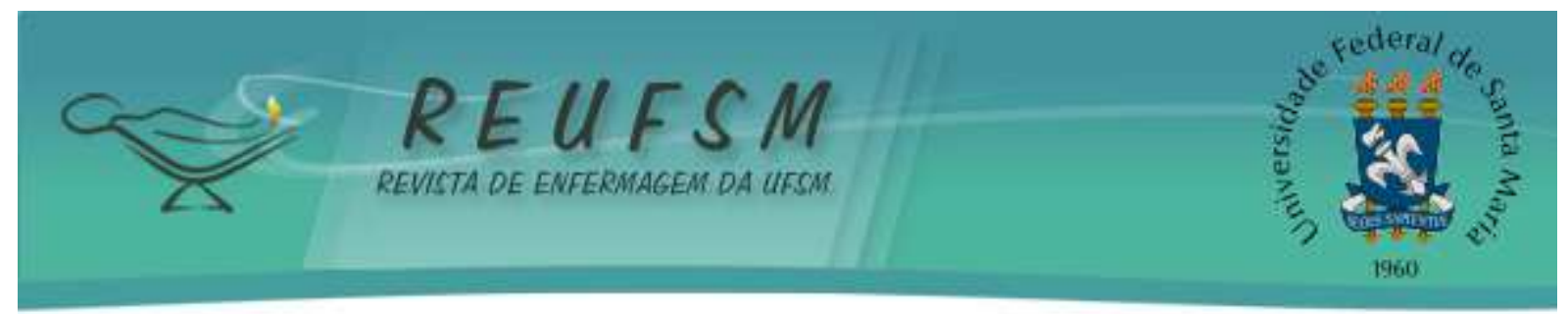

Incluíram-se todos os prontuários de mulheres que planejaram ter o parto domiciliar e iniciaram o trabalho de parto espontaneamente no domicílio, e dos respectivos RNs, não havendo perda amostral. Foram excluídos os casos em que o parto domiciliar foi contraindicado por questões de saúde materna ou neonatal. Para coleta de dados, elaborou-se um instrumento padronizado baseado em estudo semelhante conduzido, ${ }^{11}$ o qual foi reestruturado para sintetizar e direcionar os dados das três equipes, tendo variáveis do perfil sociodemográfico, clínicos obstétricos da gestação atual, do trabalho de parto no domicílio, do parto, do puerpério imediato e das condições de nascimento dos neonatos.

Para tratamento dos dados empregou-se o Statistical Package for Social Science versão 20.0, analisando-os por meio da estatística descritiva simples. Os achados foram organizados e tabulados para facilitar a análise e discussão, com base nas mais atuais evidências científicas sobre a temática.

A coleta dos dados foi realizada no mês de agosto de 2016 nos consultórios das equipes onde os prontuários e documentos estavam arquivados, após aprovação do projeto pelo Comitê de Ética em Pesquisa do Distrito Federal sob o parecer de número 1.621.558 de 27/06/2016, e Certificado de Apresentação para Apreciação Ética (CAAE) 56959816.3.0000.5553. A análise dos registros foi realizada de forma anônima, sendo atribuída uma numeração aleatória, a fim de preservar o sigilo da identificação das participantes, sendo todos os aspectos éticos envolvidos na pesquisa cumpridos conforme a Resolução n ${ }^{\circ}$ 466/12 do Conselho Nacional de Saúde.

\section{RESULTADOS}

Dos 99 prontuários analisados, 11 foram provenientes da equipe A, 53 da equipe B e 35 da equipe C. A média de idade das mulheres foi de 31,6 (DP $\pm 2,83)$ anos. A maioria, 87 $(87,9 \%)$, tinha ensino superior completo, oito $(8,1 \%)$ tinham ensino médio completo, três $(3,0 \%)$ estavam concluindo o ensino superior e uma (1,0\%) tinha ensino médio incompleto. No total, 90 (90,9\%) eram casadas ou encontravam-se em união estável e nove $(9,1 \%)$ eram solteiras ou divorciadas. Do total, $78(78,8 \%)$ exerciam alguma atividade remunerada, 18 $(18,2 \%)$ eram do lar e três $(3,0 \%)$ estudantes.

Todas as mulheres foram acompanhadas pelas equipes durante o pré-natal, das quais 45 (45,5\%) eram nulíparas e 54 (54,5\%) tinham, pelo menos, um filho. Dentre essas últimas, 33 $(61,1 \%)$ tiveram somente parto vaginal, $18(33,3 \%)$ tiveram cesarianas e três $(5,6 \%)$ tiveram ambas as experiências. Das 36 mulheres que tiveram parto vaginal, 20 (55,5\%) já haviam vivenciado o 


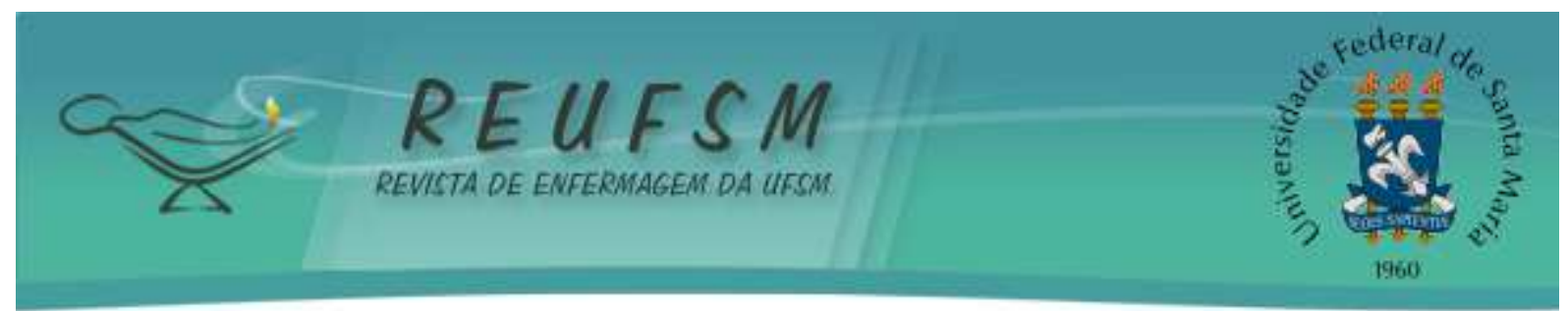

PDP, $15(41,7 \%)$ tiveram no ambiente hospitalar e uma (2,8\%) teve sua experiência anterior em centro de parto normal. Vale ressaltar que, na soma das 18 mulheres que tiveram somente cesarianas com as 45 nulíparas, totalizaram $63(63,6 \%)$ mulheres que nunca tiveram parto via vaginal.

Na tabela 1, têm-se as variáveis relacionadas à avaliação do trabalho de parto e parto no domicílio. Os parâmetros avaliados foram: frequência cardíaca fetal (FCF) (avaliada pela ausculta intermitente na fase ativa do trabalho de parto), estado das membranas no decorrer do trabalho de parto e parto, coloração do líquido amniótico das 99 mulheres, além de posição de parto e duração da fase ativa e período expulsivo das 80 mulheres que evoluíram para o parto em casa. Dessas últimas, destaca-se que em 35 (43,7\%) a expulsão fetal ocorreu na água.

Tabela 1 - Distribuição das variáveis avaliadas durante o trabalho de parto e parto no domicílio. Brasília, DF, Brasil, 2015. (N=99)

\begin{tabular}{|c|c|c|}
\hline Variável & $\mathbf{N}$ & $(\%)$ \\
\hline \multicolumn{3}{|c|}{ Frequência cardíaca fetal no decorrer do trabalho de parto } \\
\hline Sem alteração & 86 & 86,9 \\
\hline Bradicardia leve & 2 & 2,0 \\
\hline Bradicardia severa & 2 & 2,0 \\
\hline Taquicardia leve & 6 & 6,1 \\
\hline Sem registro & 3 & 3,0 \\
\hline \multicolumn{3}{|l|}{ Estado das membranas amnióticas } \\
\hline Íntegras (parto empelicado) & 3 & 3,0 \\
\hline Amniorrexe prematura & 21 & 21,2 \\
\hline Amniorrexe & 60 & 60,6 \\
\hline Amniotomia & 9 & 9,1 \\
\hline Sem registro & 6 & 6,1 \\
\hline \multicolumn{3}{|l|}{ Coloração do líquido amniótico } \\
\hline Claro & 84 & 84,8 \\
\hline Meconial & 9 & 9,1 \\
\hline Sem registro & 6 & 6,1 \\
\hline \multicolumn{3}{|l|}{ Posição de parto* } \\
\hline Semi-sentada na água, ajoelhada na água & 26 & 32,5 \\
\hline Gaskin na água, cócoras na água & 9 & 11,2 \\
\hline Gaskin, genupeitoral & 9 & 11,2 \\
\hline Cócoras, sentada na banqueta de parto & 30 & 37,5 \\
\hline Decúbito lateral, semi-sentada & 5 & 6,3 \\
\hline Em pé & 1 & 1,3 \\
\hline \multicolumn{3}{|l|}{ Duração da fase ativa até a expulsão* } \\
\hline Até 3:59 min & 36 & 45,0 \\
\hline 4 horas a 5:59 min & 23 & 28,8 \\
\hline 6 horas a $8: 59 \mathrm{~min}$ & 12 & 15,0 \\
\hline Mais de 8 horas & 9 & 11,2 \\
\hline
\end{tabular}

*N=80, mulheres que evoluíram com o parto no domicílio. 


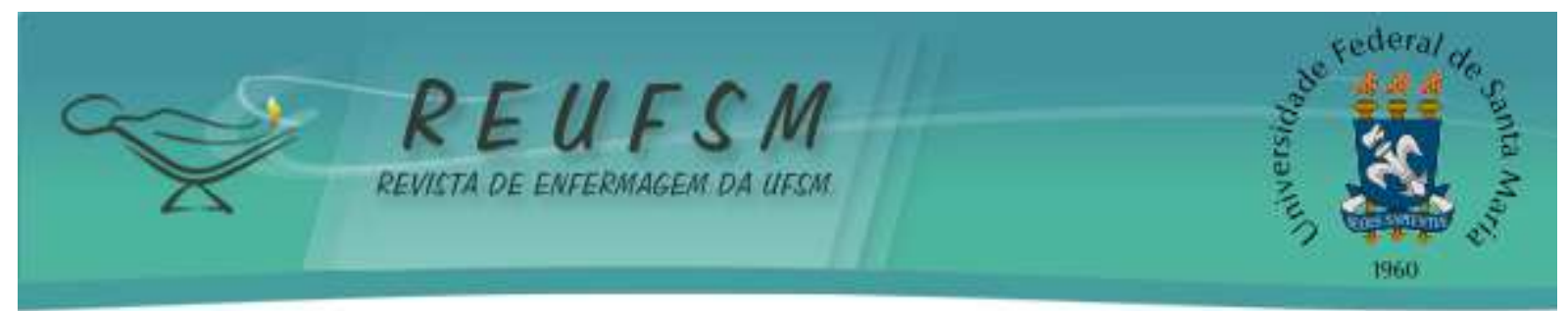

Ainda se tratando das variáveis relacionadas à avaliação do trabalho de parto, 27 $(27,3 \%)$ das mulheres assistidas tiveram o partograma preenchido. Dessas, 22 (81,5\%) apresentaram curva ascendente satisfatória de evolução do trabalho de parto e cinco $(18,5 \%)$ tiveram cruzamento, indicando a necessidade de condutas apropriadas para correção de desvios da fisiologia, sendo transferidas.

Com relação à distócia funcional e presença de acompanhantes, das 99 mulheres, cinco delas $(5,0 \%)$ não foram inseridas nessas variáveis por serem transferidas ao hospital antes do trabalho de parto ativo. Dentre as 94 incluídas, 90 (95,7\%) não tiveram alterações na dinâmica uterina, três $(3,2 \%)$ apresentaram bradissistolia e uma $(1,1 \%)$ teve taquissistolia. No que concerne aos diversos métodos não farmacológicos para alívio da dor utilizados pelas mulheres, os três predominantes foram banho de imersão em 68 mulheres (72,3\%), banho terapêutico de chuveiro em $34(36,2 \%)$ e uso da bola suíça em 14 (14,9\%). A maioria, 82 $(87,2 \%)$ teve acompanhante durante o processo de trabalho de parto e nascimento no domicílio, e em 12 (12,8\%) prontuários não haviam esse registro. Quanto aos acompanhantes presentes, os mais frequentes foram o companheiro em 78 prontuários $(83,0 \%)$, a doula em 29 $(30,8 \%)$ e os filhos em $27(28,7 \%)$.

Dos 99 documentos analisados, $80(80,8 \%)$ mulheres tiveram parto domiciliar e 19 $(19,2 \%)$ hospitalar após transferências. A tabela 2 indica as variáveis dos partos que evoluíram em casa quanto à avaliação do período pós-parto imediato e compreendem as condições do períneo, o uso de medicação pós-parto, contato pele a pele, amamentação na primeira hora, peso do $\mathrm{RN}$, índice de agpar no primeiro e quinto minuto de vida, e classificação dos RNs quanto à idade gestacional. Ressalta-se que não houve nenhum caso de episiotomia realizada na amostra estudada.

Tabela 2 - Distribuição das variáveis avaliadas durante o pós-parto imediato no domicílio. Brasília, DF, Brasil, 2015. (N=80)

\begin{tabular}{lcc}
\hline Variável & $\mathbf{N}$ & $(\boldsymbol{\%})$ \\
\hline Condições do períneo & 59 & 73,8 \\
$\quad$ Sem trauma ou sem sutura & 21 & 26,2 \\
$\quad$ Laceração $1^{\circ}$ ou $2^{\circ}$ grau suturada & & \\
Medicação no pós-parto imediato & 64 & 80,0 \\
$\quad$ Nenhuma & 16 & 20,0 \\
$\quad$ Ocitocina & & \\
Contato pele a pele & 70 & 87,5
\end{tabular}




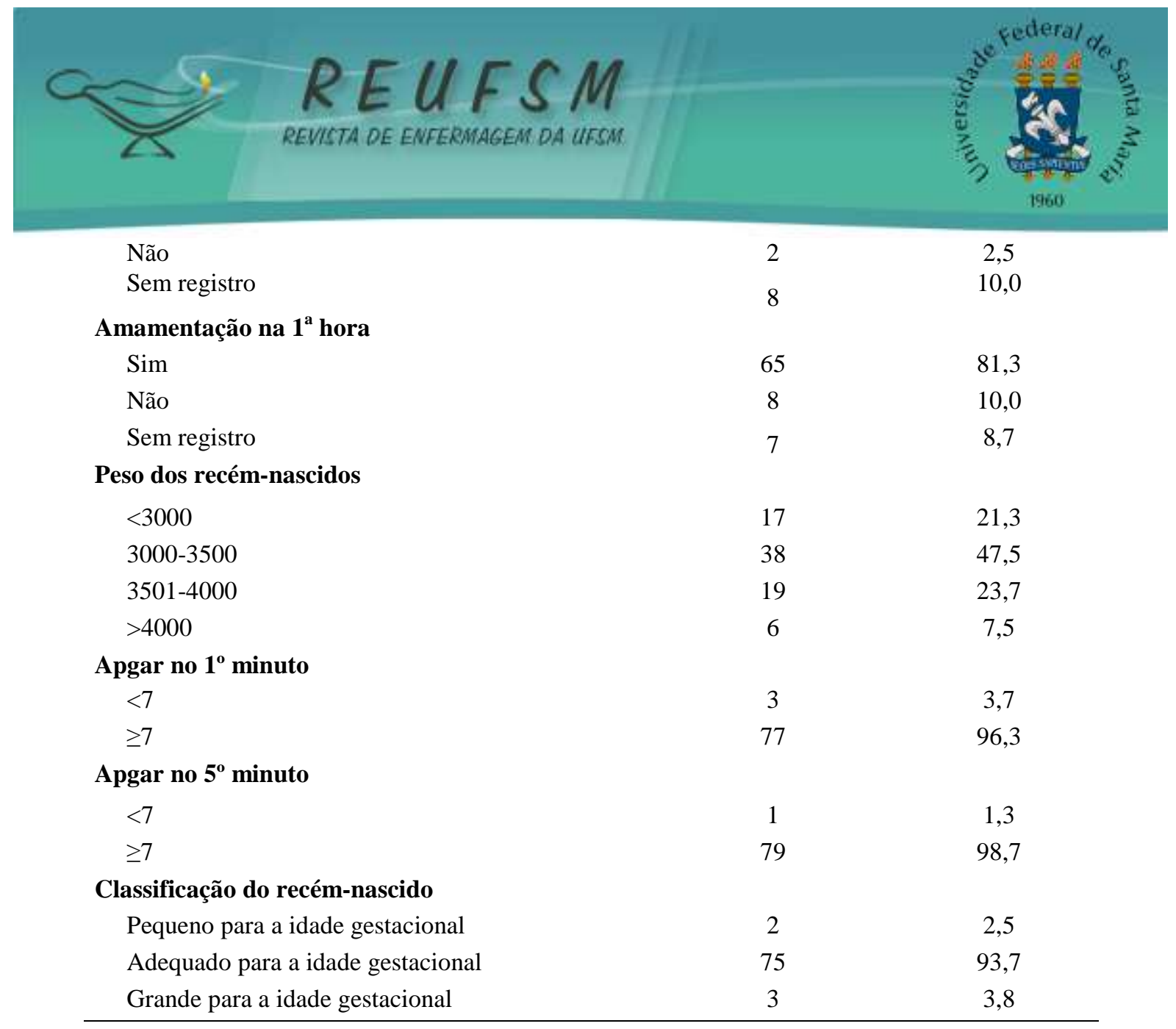

A maioria $77(96,3 \%)$ não apresentou intercorrências no pós-parto imediato no domicílio e três $(3,7 \%)$ evoluíram com alguma intercorrência, das quais: uma puérpera apresentou hemorragia pós-parto que foi resolvida de imediato, sem necessidade de transferência, e duas corresponderam a transferências pós-parto para instituição hospitalar, as quais serão descritas adiante.

Dos 99 neonatos, 19 (19,2\%) nasceram em hospitais após transferência de suas mães antes do parto, não sendo possível avaliar devido à ausência de registro nos prontuários das equipes. Os outros $80(80,8 \%)$ que nasceram no domicílio, $100 \%$ tiveram clampeamento do cordão umbilical tardio, após o primeiro minuto de vida. Apenas dois (2,5\%) dos RNs necessitaram de ventilação com pressão positiva para iniciar e/ou manter movimentos respiratórios efetivos, sem intercorrências nas horas subsequentes de vida, permanecendo, assim, no domicílio.

Somando-se as transferências realizadas desde a fase inicial do trabalho de parto até o pós-parto, verificou-se que $21(21,2 \%)$ mulheres foram transferidas, sendo que todas internaram em hospital particular, 17 (17,2\%) realizaram cesariana e duas (2,0\%) evoluíram para parto vaginal conduzido. Em relação ao profissional que prestou atendimento na 


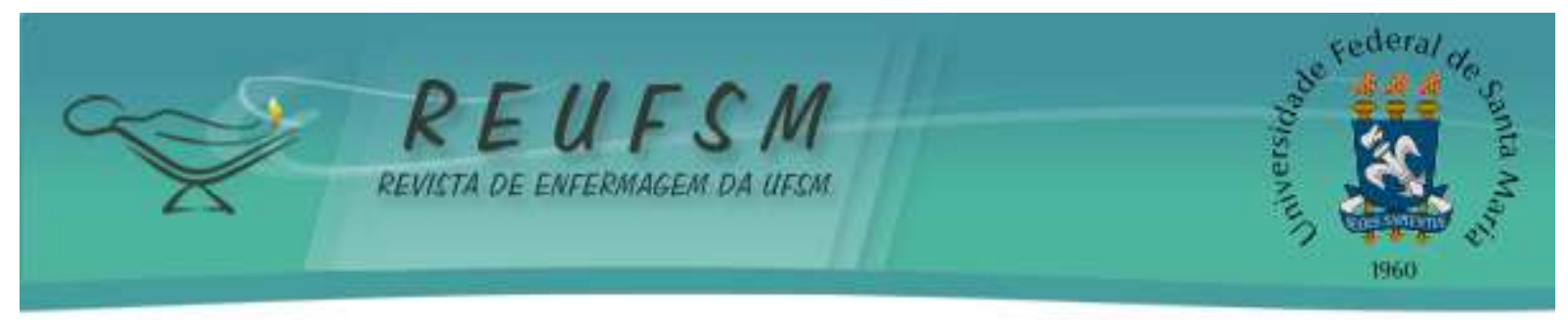

instituição, $12(57,1 \%)$ foram recebidas por médicos plantonistas e nove $(42,9 \%)$ por médicos particulares que estavam de sobreaviso. Dentre os motivos, cinco $(23,8 \%)$ parturientes foram removidas antes do trabalho de parto ativo e apresentavam bolsa rota acima de 16 horas. Duas delas $(9,5 \%)$ decidiram ir para o hospital, outras duas $(9,5 \%)$ apresentaram frequência cardíaca fetal (FCF) alterada e uma (4,8\%) apresentou elevação da pressão arterial.

Das $14(66,7 \%)$ parturientes removidas na fase ativa do trabalho de parto, cinco $(23,8 \%)$ foram por parada de progressão da descida fetal, cinco $(23,8 \%)$ por alteração da FCF, três $(14,2 \%)$ por parada da dilatação cervical e uma $(4,8 \%)$ por distócia funcional. Já no pós-parto imediato, duas $(9,5 \%)$ foram removidas, sendo uma $(4,8 \%)$ por retenção placentária associada à hemorragia pós-parto controlada e outra $(4,8 \%)$ também por retenção placentária juntamente com seu RN que apresentava Síndrome de Down não diagnosticada no pré-natal, sendo este o único $(1,2 \%)$ RN transferido. Todas as remoções foram bem-sucedidas e realizadas em tempo oportuno, sem risco de vida ou de internação prolongada para o binômio.

Quanto à internação em unidade de terapia intensiva (UTI), nenhuma das mulheres necessitou de internação. Apenas um $(4,8 \%)$ RN foi para UTI neonatal para profilaxia com antibiótico devido à rotina hospitalar para tempo de bolsa rota maior que 24 horas, recebendo alta em seguida, sem complicações.

\section{DISCUSSÃO}

O perfil sociodemográfico vai ao encontro de outros estudos nacionais e internacionais, os quais apontam predomínio de uma faixa etária entre 28-32 anos, casadas ou que viviam com parceiro, sendo a maioria com ensino superior completo ou em curso, e exerciam atividade remunerada. $^{2,11,13-17}$ É possível associar esse perfil à maior acessibilidade, à informação e poder aquisitivo elevado, pois a contratação desse tipo de serviço nos grandes centros urbanos se restringe ao sistema privado, exceto em Belo Horizonte, onde se tem inovado com um modelo de assistência ao PDP vinculado ao Sistema Único de Saúde (SUS), iniciativa que estimula o parto domiciliar. ${ }^{16}$ No entanto, essa não é a realidade nas outras capitais brasileiras em que esse serviço é exclusivamente privado, restringindo o acesso à população com condições sociais menos favorecidas, a qual não tem o PDP como opção e é condicionada a receber a assistência tradicional no hospital ou em casas de parto. 


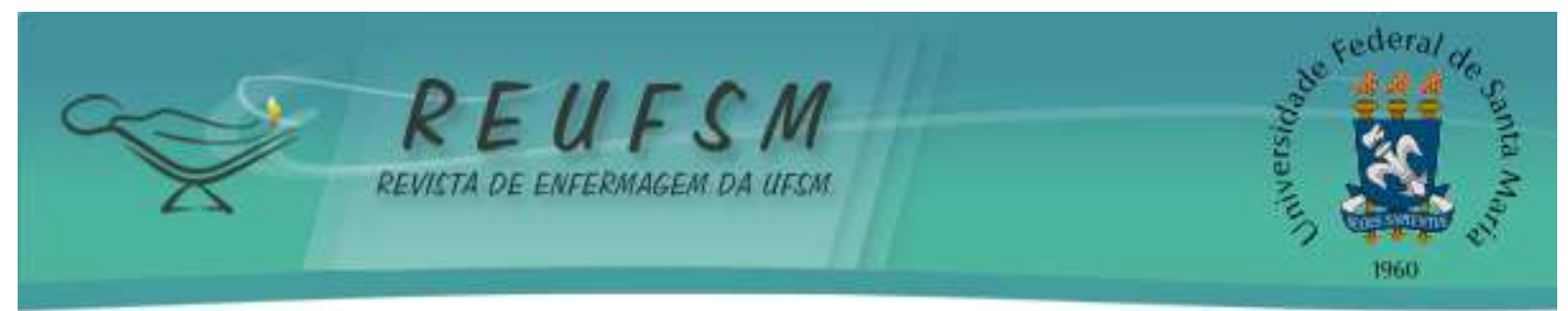

A taxa de mulheres com cesárea prévia foi menor do que a encontrada na literatura de $53,1 \%$. A experiência negativa em partos anteriores instiga as mulheres a buscarem outros meios para terem seus filhos e acreditam ser o domicílio o melhor lugar. Apesar da cesárea prévia não contraindicar o parto normal, é necessária uma avaliação cuidadosa para elegibilidade do PDP, além de uma assistência ainda mais rigorosa durante o trabalho de parto e parto. ${ }^{13,18}$ Por outro lado, das que tiveram parto vaginal, grande parte teve um PDP anterior, o que revelou o interesse em vivenciar novamente uma experiência positiva. Estudos internacionais apresentam maior segurança para PDP em segundo parto vaginal comparado ao primeiro., ${ }^{3,18}$ Com isso, é indispensável investir em incentivos para o parto normal em primigestas na intenção de prevenir uma primeira cesárea e reduzir os riscos em futuras gestações.

O padrão não tranquilizador da FCF apresentou valor próximo em um estudo similar. ${ }^{11}$ Vale destacar que este foi um dos principais motivos de transferência na amostra estudada e, de forma geral, esse desvio da fisiologia é identificado precocemente a partir de uma monitorização atenta e frequente. Quanto à presença de líquido amniótico meconial, o valor apresentado é maior que em outros estudos, contudo sem complicações perinatais. ${ }^{11,19}$ Em relação à amniotomia, os resultados revelaram um valor próximo ao achado na literatura, sendo uma intervenção realizada devido ao desvio na curva do partograma. ${ }^{11,18-19}$ Essa prática, geralmente é usada para acelerar o trabalho de parto e pode desencadear uma cascata de eventos iatrogênicos, porém quando aplicada com uma indicação respaldada no gráfico do partograma, favorece a evolução para um parto vaginal.

Os achados revelaram um tempo de trabalho de parto ativo e expulsivo semelhante ao de outro estudo. ${ }^{11}$ Vale lembrar que $63,6 \%$ das mulheres correspondiam ao primeiro parto vaginal, e que esse tempo foi menor do que o descrito na literatura, cerca de 10 horas para o primeiro parto. $^{20}$ Mostrou-se, assim, que o domicílio oferece condições ideais para o relaxamento da mulher e o nascimento fisiológico. A ambiência é considerada um fator importante na evolução do trabalho de parto. A garantia da privacidade, baixa luminosidade, silêncio, além de relação de vínculo construída entre a família e a equipe são fatores que fortalecem a ideia de segurança entre os casais, o que diminui a tensão e a ansiedade, e favorece a liberação hormonal e a fisiologia do parto. ${ }^{17,21}$

O predomínio de partos verticais e na água, coincidem com os de outros estudos. ${ }^{4,11}$ As posições verticalizadas assumidas no trabalho de parto e parto são consideradas como 


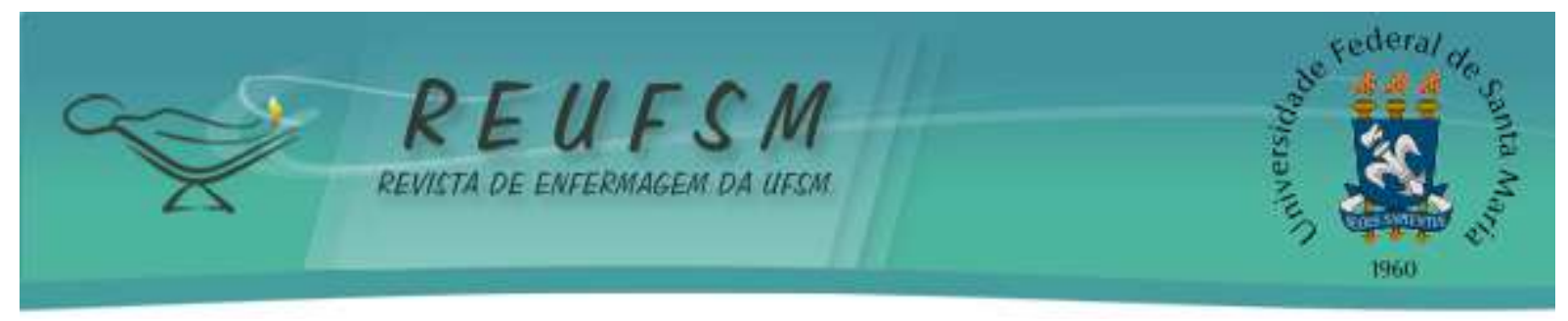

facilitadoras na evolução fisiológica por favorecer a descida do feto pela pelve e amenizar a sensação de dor. O parto na água é idealizado pelas mulheres que planejam parir em casa, sendo associado à suavização do nascimento para o RN e ao relaxamento ocasionado com a redução da dor, fato este que confirma a utilização da água, seja por imersão ou aspersão, como método não farmacológico mais utilizado para alívio da dor. Outras pesquisas também apontam esses métodos como os mais escolhidos pelas mulheres. ${ }^{14,22}$

Os resultados obtidos se assemelham aos encontrados em outros estudos em que todas as mulheres tiveram a presença de, pelo menos, um acompanhante, predominando a presença do companheiro. Vários autores ressaltam a importância da participação da família no processo do nascimento, por serem pessoas especiais e de escolha da mulher, que transmitem confiança, conforto e encorajamento. ${ }^{11,15,17,22-24}$

No que tange às boas práticas recomendadas no parto, além das descritas, como a participação da família no processo, intervenções criteriosas e justificadas com o uso do partograma, ambiência, posições livres à escolha da mulher, entre outras, tem-se o uso restrito da episiotomia. As evidências científicas têm elucidado os danos provenientes do uso rotineiro da episiotomia e reforçado os benefícios e a segurança em não utilizar essa prática. ${ }^{6}$ Outros estudos já apresentam uma redução do procedimento de até sete vezes em PDP comparando ao parto hospitalar, e associam esse local de nascimento com uma menor probabilidade de laceração perineal de terceiro ou quarto grau, assemelhando aos resultados apresentados. $^{11,13,21}$ Os dados reforçam que a episiotomia é uma prática desnecessária, sugerindo sua abolição na assistência ao parto.

Os desfechos relacionados aos RNs são condizentes com os da literatura. Evidenciaramse dados favoráveis, maiores índices de contato pele a pele, amamentação na primeira hora, índices de apgar maiores que sete no primeiro e quinto minuto de vida, e o clampeamento tardio do cordão umbilical em todos os bebês. ${ }^{5,13,19,21,25}$ Associam ainda o PDP assistido por profissionais qualificados com redução no uso de cuidados neonatais especializados, como terapia de oxigênio nas primeiras 24 horas de vida, ressuscitação, internações hospitalares e há redução em traumas no nascimento. ${ }^{13}$

Há quem questione o PDP, justificando o alto risco de complicações e o tempo gasto até chegar a uma instituição. ${ }^{23}$ Porém, algumas evidências constataram menor risco de desfechos maternos adversos em PDP, citando a hemorragia pós-parto como exemplo, o que corrobora 


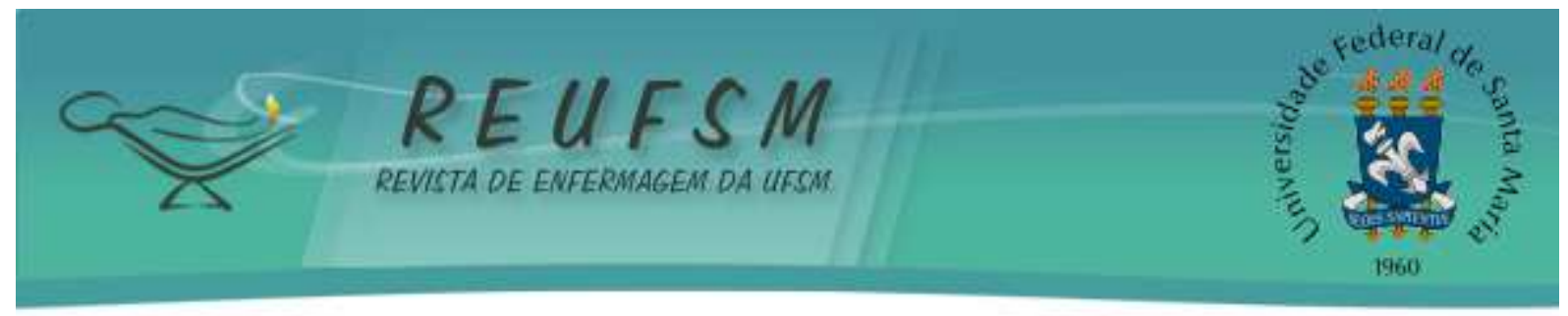

com os achados dessa investigação, com uma taxa mínima de intercorrências. ${ }^{13,19,21,25}$ Esse modelo de assistência exige um planejamento prévio e uma avaliação da distância mínima entre a residência da mulher e o hospital, assim como a condução nos primeiros sinais de desvio da fisiologia, fatores que são considerados indispensáveis para os bons resultados descritos.

$\mathrm{Na}$ análise das transferências maternas, observou-se uma taxa superior à descrita na literatura, apresentando semelhanças no que tange aos motivos de remoção intraparto e pósparto imediato. ${ }^{5,13,19}$ Pesquisa internacional aponta uma taxa de $45 \%$ de transferência para mulheres que planejavam ter o primeiro filho via vaginal no domicílio, valor maior que o apresentado. $^{2}$ Os resultados encontrados não tiveram caráter comparativo entre as equipes, sendo apresentados apenas os resultados gerais, porém, destaca-se que uma equipe pode apresentar taxas menores que a outra, assim como divergência nos motivos de transferências, o que repercute no aumento dessa taxa na amostra apresentada.

Os desfechos positivos apresentados devem-se ao respeito à fisiologia e ao uso restrito de intervenções. Autores destacam que para atingir tais resultados, é necessária uma avaliação rigorosa na elegibilidade das mulheres a serem acompanhadas e a presença de um profissional qualificado. ${ }^{17,19,22-23,25}$ Destaca-se a necessidade da enfermeira obstétrica estar em constate atualização quanto às recomendações e evidências atuais para embasar sua atuação durante o pré-natal, principalmente na elegibilidade, o trabalho de parto, o parto e nascimento, promovendo, assim, uma assistência segura.

Como observado em um estudo multicêntrico comparativo entre a assistência hospitalar obstétrica, casas de partos e partos domiciliares, a assistência domiciliar ao parto não acarretou riscos ao binômio em comparação aos partos ocorridos em outros locais, desde que respeitadas as gestações de risco habitual. ${ }^{3}$ Os resultados apontados reiteram que a assistência conduzida pelas enfermeiras obstétricas é segura; e que a identificação de qualquer alteração foi realizada precocemente, com uma assistência de qualidade e com segurança na remoção. Ademais, podese inferir que não houve intercorrências que culminaram com desfechos negativos ao binômio.

\section{CONCLUSÃO}

Verificou-se como limitação, a ausência de alguns registros nos prontuários, tais como os dados dos binômios transferidos para o hospital e os resultados do pós-parto tardio, que 


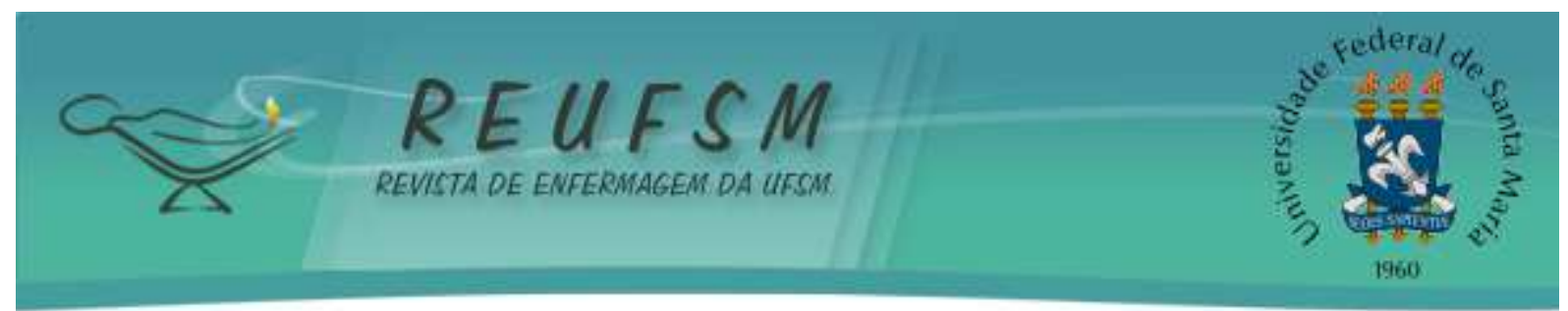

teriam ampliado a perspectiva das evidências apontadas, tais como presença de icterícia, problemas com a amamentação, dor perineal, infecções, entre outros.

Salienta-se a atuação das enfermeiras obstétricas, pois empregaram e incentivaram as boas práticas de atenção ao parto e nascimento recomendadas, tais como a adoção de posições facilitadoras para o parto, baixas taxas de lacerações perineais e de necessidade de perineorrafia, realização de clampeamento tardio do cordão, contato pele a pele, amamentação na primeira hora de vida, e a participação da família. Isso repercutiu em um tempo de evolução do trabalho de parto e nascimento consideravelmente rápido, escores desejáveis de apgar, além de intervenções realizadas em tempo oportuno com transferências para o hospital sem complicações, e com bons resultados para genitora e neonato.

Como contribuição, os resultados apresentados permitem inferir o PDP como uma opção segura, viável e de qualidade para a família vivenciar esse processo. Os dados permitem, ainda, ampliar as discussões sobre a temática e beneficiar às mulheres que fazem essa escolha no enfrentamento dos desafios envolvidos.

Conclui-se que os resultados positivos são resultantes da assistência cautelosa e segura prestada pelas enfermeiras obstétricas, valorizando a atuação dessas profissionais, fortalecendo sua autonomia e contribuindo para o seu reconhecimento neste cenário de parto no Brasil.

Destaca-se a necessidade de estudos que divulguem dados dessa modalidade de assistência ao parto, comprovando seus resultados com bases científicas e incentivando o emprego dessa modalidade tanto no sistema privado quanto no sistema público de saúde, o que permitiria o acesso universal à população.

\section{REFERÊNCIAS}

1. Tura PF. Grito primal - o parto natural domiciliar como performance como arte. Rev Poiésis [Internet]. 2014 [acesso em 21 mar 2018];1(24):215-26. Disponível em: http://www.poiesis.uff.br/p24/pdf/p24-artigo-3-paula-ferreira.pdf.

2. Sanfelice CFO, Abbud FSF, Pregnolatto OS, Silva MG, Shimo AKK. Do parto institucionalizado ao parto domiciliar. Rev Rene. 2014;15(2):362-70.

3. Sandall J. Birthplace in England Research - implications of new evidence. J Perinat Educ [Internet]. 2013 [acesso em 20 jan 2016];22(2):77-82. Disponível em: http://www.ncbi.nlm.nih.gov/pmc/articles/PMC3647739/\#.

4. Sanfelice CFO, Shimo AKK. Parto domiciliar: avanço ou retrocesso? Rev Gaúcha Enferm. 2014;35(1):157-60. 


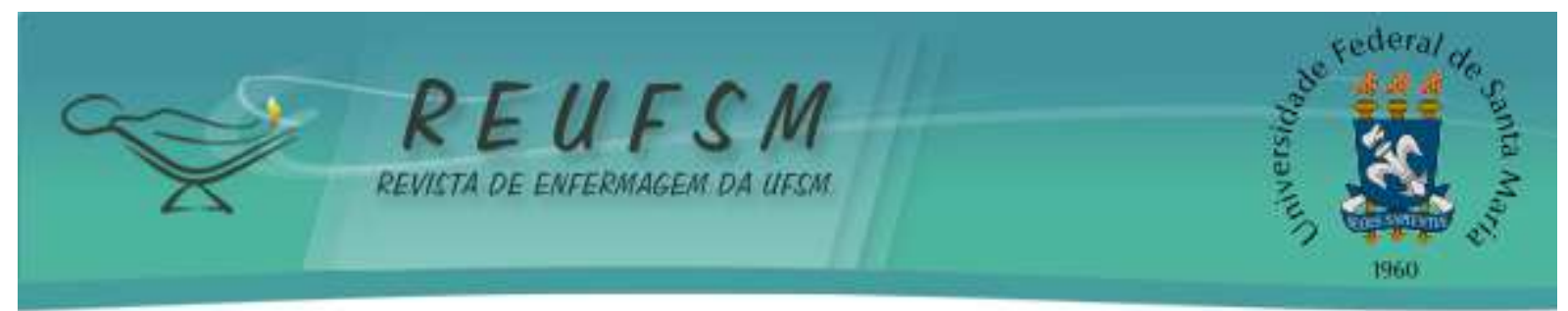

5. Doherty D, Nathan L, Hutchinson M, Somerville S, Hauck Y, Hornbuckle J. Planned home and hospital births in Western Australia: 2002-2013. J Paediatr Child Health [Internet]. 2017 [acesso em 09 mar 2018];53(S2):27. Disponível em: http://onlinelibrary.wiley.com/doi/10.1111/jpc.13494_72/full.

6. Brasil. Ministério da Saúde. Secretaria de Ciência, Tecnologia e Insumos Estratégicos Departamento de Gestão e Incorporação de Tecnologias em Saúde. Diretrizes Nacionais de Assistência ao Parto Normal - versão resumida. Brasília (DF): Ministério da Saúde; 2017.

7. Brasil. Ministério da Saúde. Diretrizes de atenção à gestante: a operação cesariana [Internet]. Brasília (DF): Ministério da Saúde; 2016.

8. Brasil. Ministério da Saúde. Agência Nacional de Saúde Suplementar. Atualização das taxas de partos na saúde suplementar: SIP/ANS. Brasília (DF): ANS; 2016.

9. Departamento de Informática do Sistema Único de Saúde (DATASUS). Sistema de Informação sobre Nascidos Vivos - SINASC. Local de nascimento, domicílio, ano 2006 a 2016. Atualizado em 2016 [internet]. Brasília (DF): Ministério da Saúde; 2016 [acesso em 09 mar 2018]. Disponível em: http://tabnet.datasus.gov.br/ cgi/tabcgi.exe?sinasc/cnv/nvuf.def.

10. Souza RM, Soares LS, Quitete JB. Home parturition: power to feminine nature and a challenge for the obstetric nurse. J Res Fundam Care [Internet]. 2014 [acesso em 21 mar 2018];6(1):118-31.

Disponível

em: https://docs.google.com/viewerng/viewer?url=http://www.seer.unirio.br/index.php/cuidadofu ndamental/article/viewFile/2260/pdf_1099.

11. Koettker JG, Bruggemann OM, Knobel R. Resultado maternos dos partos domiciliares planejados assistidos por enfermeiras da Equipe Hanami no sul do Brasil, 2002-2012. Texto \& Contexto Enferm [Internet]. 2017 [acesso em 09 mar 2018];26(1):e3110015. Disponível em: http://www.scielo.br/pdf/tce/v26n1/pt_0104-0707-tce-26-01-3110015.pdf.

12. Mattos DV, Vandenberghe L, Martins CA. O enfermeiro obstetra no parto domiciliar planejado. Rev Enferm UFPE [Internet]. 2016 [acesso em 20 jan 2017];10(2):568-75. Disponível em: http://www.revista.ufpe.br/revistaenfermagem/ index.php/revista/article/viewFile/8587/pdf_9599.

13. Cheyney M, Bovbjerg M, Everson C, Gordon W, Hannibal D, Vedam S. Outcomes of Care for 16,924 Planned home births in the United States: the midwives alliance of North America statistics project, 2004 to 2009. J Midwifery Womens Health [Internet]. 2014 [acesso em 09 mar 2018]. Disponível em: http://sci-hub.tw/10.1111/jmwh.12172.

14. Sanfelice CFO, Shimo AKK. Boas práticas em partos domiciliares: perspectivas de mulheres que tiveram experiência de parto em casa. Rev Eletrônica Enferm [Internet]. 2016 [acesso em 20 dez 2016];18:e1159. Disponível em: https://www.revistas.ufg.br/fen/article/view/31494/21363.

15. Castro CM. Os sentidos do parto domiciliar planejado para mulheres do município de São Paulo, São Paulo. Cad Saúde Colet [Internet]. 2015 [acesso em 13 jan 2016];23(1):69-75. Disponível em: http://www.scielo.br/pdf/cadsc/v23n1/1414-462X-cadsc-23-01-00069.pdf.

16. Feyer ISS, Monticelli M, Knobel R. Perfil de casais que optam pelo parto domiciliar assistido por enfermeiras obstétricas. Esc Anna Nery Rev Enferm [Internet]. 2013 [acesso em 10 dez 2015];17(2):298-305. Disponível em: http://www.scielo.br/pdf/ean/v17n2/v17n2a14.pdf. 


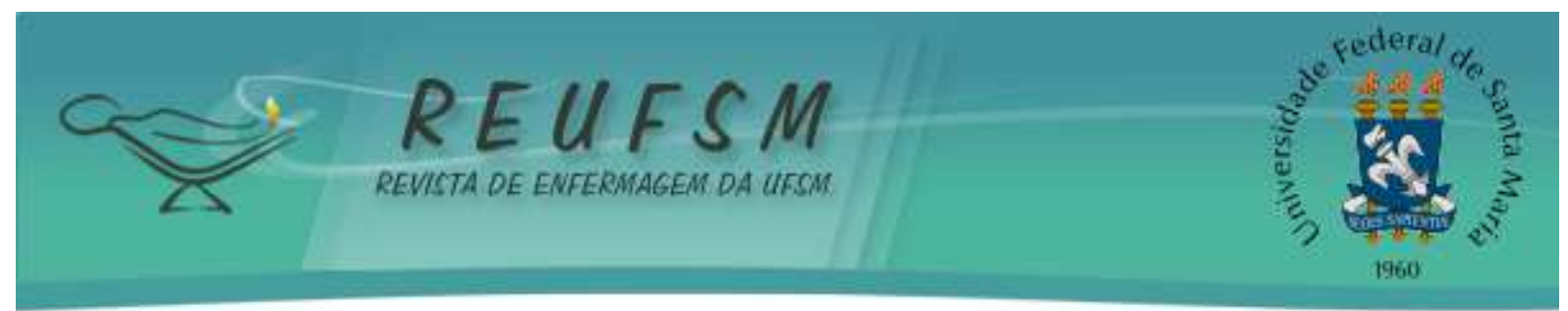

17. Collaço VS, Santos EKA, Souza KV, Alves HVA, Zampieri MF, Gregório VRP. O significado atribuído pelo casal ao parto domiciliar planejado, assistido pelas enfermeiras obstétricas da equipe Hanami. Texto \& Contexto Enferm [Internet]. 2017 [acesso em 08 mar 2018];26(2):e603015. Disponível em: http://www.scielo.br/pdf/tce/v26n2/pt_0104-0707-tce-2602-e6030015.pdf.

18. Hutton EK, Cappelletti A, Reitsma AH, Simioni J, Horne J, McGregor C, et al. Outcomes associated with planned place of birth among women with low-risk pregnancies. CMAJ [Internet]. 2016 [acesso em 09 mar 2018];188(5):E80-90. Disponível em: http://www.cmaj.ca/content/cmaj/early/2015/12/22/cmaj.150564.full.pdf.

19. Koettker JG, Bruggemann OM, Dufloth RM. Partos domiciliares planejados assistidos por enfermeiras obstétricas: transferências maternas e neonatais. Rev Esc Enferm USP [Internet]. 2013 [acesso em 01 out 2015];47(1):15-21. Disponível em: http://www.scielo.br/pdf/reeusp/v47n1/a02v47n1.pdf.

20. Cunningham FG, Leveno KJ, Bloom SL, Spong CY, Dashe JS, Hoffman BL, et al. Obstetrícia de Williams. 24º ed. Porto Alegre: AMGH; 2016.

21. Zielinski R, Ackerson K, Low LK. Planned home birth: benefits, risks, and opportunities. Int J Womens Health Wellness [Internet]. 2015 [acesso em 27 set 2016];7:361-77. Disponível em: https://www.dovepress.com/planned-home-birth-benefits-risks-and-opportunities-peerreviewed-article-IJWH.

22. Feyer ISS, Monticeli M, Boehs AE, Santos EKA. Rituais de cuidado realizados pelas famílias na preparação para a vivência do parto domiciliar planejado. Rev bras enferm. 2013;66(6):879-86.

23. Sanfelice CFO, Shimo AKK. Parto domiciliar: compreendendo os motivos dessa escolha. Texto \& Contexto Enferm [Internet]. 2015 [acesso em $20 \mathrm{dez}$ 2016];24(3):875-82. Disponível em: http://www.scielo.br/pdf/tce/v24n3/pt_0104-0707-tce-24-03-00875.pdf.

24. Frank TC, Pelloso SM. A percepção dos profissionais sobre a assistência ao parto domiciliar planejado. Rev Gaúcha Enferm. 2013;34(1):22-9.

25. Jonge A, Geerts CC, Van Der Goes BY, Mol BW, Buitendijk SE, Nijhuis JG. Perinatal mortality and morbidity up to 28 days after bith among 743070 low-risk planned home and hospital biths: a cohort study based on three merged national perinatal databases. BJOG [Internet]. 2015 [acesso em 07 mar 2018];122(5):720-8. Disponível em: https://www.ncbi.nlm.nih.gov/pubmed/25204886.

Data de submissão: 15/08/2017

Data de aceite: 26/03/2018

Autor correspondente: Simone Silva dos Santos

Endereço: Módulo AC 401, lote 48, Residencial Santa Maria, Brasília, Distrito Federal, Brasil. CEP: 72501-124

Email: eo.simonesilva@gmail.com 